\title{
面向一流课程多维度推进无机化学实验课程改革与实践
}

马艺, 魏灵灵, 李淑妮, 薛东旭, 徐玲, 张伟强, 焦桓 ${ }^{*}$

陕西师范大学化学化工学院, 西安 710119

摘要: 详细总结陕西师范大学化学化工学院在无机化学实验课程改革的实施情况, 从教学内容、考核模式、教学平 台、课程思政以及教学总结等方面进行阐述, 并通过典型案例 “二氧化碳分子量的测定” 介绍了各项改革内容如何 具体实施，希望为相关课程的改革提供借鉴。

关键词：无机化学实验；Blackborad教学平台；课程思政；虚拟仿真实验 中图分类号: G64; O6

\section{Promoting the Reform and Practice of Inorganic Chemistry Laboratory Course in Multi-Dimensions for First-Class Course}

Yi Ma, Lingling Wei, Shuni Li, Dongxu Xue, Ling Xu, Weiqiang Zhang, Huan Jiao *

School of Chemistry \& Chemical Engineering, Shaanxi Normal University, Xi'an 710119, China.

Abstract: In this paper, the reform implementation of inorganic chemistry laboratory at the College of Chemistry and Chemical Engineering of Shaanxi Normal University was summarized. The reform content included teaching content, assessment mode, teaching platform, course ideology and politics and teaching summary. The typical case "Determination of molecular weight of carbon dioxide" was used to elaborate how the reform was implemented in detail. We hope this paper can provide useful information for the reform of related courses.

Key Words: Inorganic chemistry laboratory; Blackborad teaching platform;

Ideological and political education of curriculum; Virtual simulation experiment

\section{1 前言}

作为教育部直属的师范类本科院校, 我校一直以来以师范专业卓越教师培养、非师范专业拔尖 创新人才培养为目标, 为祖国不断输送具有西部红烛精神的各级各类优秀专业人才。化学化工学院 作为我校 “一头两翼”一流学科中的重点单位, 在人才培养中肩负着重要职责。无机化学实验是化 学、化工、材料、生科、食品等专业大一新生入校的第一门专业必修实验课。其在整个化学教学中 占有举足轻重的地位, 肩负着引导学生进入、适应大学学习, 激发学生学习兴趣以及培养学习习惯 的关键性作用, 同时也发挥着衔接中学与大学教育, 训练学生思维模式转变的作用。因此, 该课程 的持续改革创新是提升整体化学教学质量的基石。

近年来, 我院依托陕西师范大学国家级化学实验教学示范中心、国家级化学虚拟仿真实验教学 中心对无机化学实验课程的软件及硬件方面进行了多方位的改革。例如, 2010年起对实验考核方式 和教学方法进行变革, 逐步形成了过程考核模式; 2013年以来, 教学团队围绕无机化学实验改革和

收稿: 2021-06-17; 录用: 2021-07-25; 网络发表: 2021-11-08

“通讯作者, Email: jiaohuan@snnu.edu.cn

基金资助：陕西省教改重点项目; 陕西师范大学教学改革综合研究项目(19JG30, 21JG03, 21JG01) 
创新进行教学研究, 撰写发表了多篇教学研究论文; 2015年教学团队组织编写了由高等教育出版社 出版的《无机化学实验》和配套的《无机化学实验手册》; 2016年将Blackboard (Bb)教学平台引入教 学过程; 2018年尝试进行课程思政建设；2019年及2020年将虚拟仿真实验引入教学内容。通过多方 位的改革措施使无机化学实验教学效果和手段都得到了极大的提升。本文将围绕以上改革内容, 对 改革实施方法、实施效果、典型实验教学过程等内容进行详细阐述, 为相关课程的改革提供有力借 鉴。

\section{2 课程改革主要方向}

为实现无机化学实验一流课程建设, 使其成为培养优秀化学人才的肥沃土壤, 改革实施必须具 有多元化、多角度的特点 ${ }^{[1,2]}$ 。因此, 我们的改革方向包含了教学内容、考核模式、教学平台、课程 思政以及教学总结等多方面, 下面将对这几方面进行具体阐述。

\section{1 教学内容}

无机化学实验是一门运用无机化学理论知识进行实验技能操作训练的课程。其教学内容与无机 化学紧密相关但又不拘泥于教材。针对无机化学实验内容老旧、脱离实验技术前沿发展等问题, 我 们对教学内容进行了梳理和调整。在保留经典操作实验的基础上增加了创新实验、虚拟仿真实验等 内容。针对实验内容不同划分为四部分:

(1) 基础操作类实验。该类实验占比约 $35 \%$, 实验内容主要保留历年教材中的经典实验, 例如粗 食盐的提纯、硝酸钾的制备和提纯, 此类实验以训练无机化学基本操作为目标, 训练内容包括药品 的称量、反应的加热、蒸发结晶、抽滤、离心等。

(2) 元素化合物的性质实验。该类实验占比约 $35 \%$, 与无机化学理论课程的元素化学部分联系最 为紧密, 例如 “氧、硫、氮、磷” “铁、钴、镍”, 以各个元素的鉴定方法、分离方法、特征反应为 考查目标。

(3) 综合类实验。该类实验占比约 $15 \%$, 以无机物的合成及组成或性质测试为教学内容, 例如三 草酸合铁酸钾的制备及组成测定、多碘盐的制备及分析, 该类实验以培养学生无机化合物的合成以 及性质分析的综合能力为主要目的。同时, 我们还注意将历年化学实验竞赛实验引入教学, 培养学 生在实验过程中独立分析问题、解决问题的能力, 在日常教学中为各级各类学生实验和学术竞赛做 好准备。

(4) 创新类实验。该类实验占比约 $15 \%$, 实验内容主要选取科研领域具有重大理论和应用意义的 典型实验, 例如氧化性石墨烯的制备、化学共沉淀法制备 $\mathrm{K}_{2} \mathrm{GeF}_{6}: \mathrm{Mn}^{4+}$ 等。该类实验紧跟科技前沿, 对实验背景知识的学习颇为重要, 为学生了解科学动态发展搭建桥梁, 培养学生的创新精神以及关 注科研动态的学习习惯。

调整后的实验教学内容既能保证学生掌握和巩固无机化学实验基本技能、基础理论和基本知识, 又能提升学生的综合实验能力以及探索创新能力。创新类实验的内容将根据科技前沿不断更新, 打 造成低年级学生了解、接触科技前沿的窗口。

\section{2 考核模式}

实验课与理论课不仅在教学形式方面存在着较大差别, 其考核方式也应根据课程的学习特点加 以区别。教学团队经过多年摸索, 逐步形成了突出 “过程化” 考核, 辅之以根据实验内容设计考核 办法的差异化实验考核模式。例如, 无机化学实验(上)的教学内容大多为制备实验, 每个实验均有可 量化的产品指标和明确的实验操作考查点, 因此该学期实验成绩以平时成绩为主要依据。而无机化 学实验(下)大部分内容为性质实验, 其操作简单, 且没有可量化的产品, 学生在实验过程中区分度较 低, 因此, 该学期的实验成绩评估中, 考试实验占比份额较大。此外, 为了更好地考查学生对基础 知识、基本理论的掌握程度, 我们针对每个实验设计了小测验, 该测验成绩也纳入到期末成绩当中。 而对于创新实验, 不仅考查学生的操作水平和产品指标, 我们还通过学生撰写的小论文考查对创新 
实验相关背景、知识的了解情况, 并通过该过程训练学生查阅资料、分析资料、整合知识的能力, 以 提升其自我学习的综合素质。

\section{3 教学平台}

在现代多媒体技术、通讯技术飞速发展的今天, 知识的获取和传播途径已经从线下的实体课堂、 实验室扩展至多种线上模式, 不仅包含了各类教学平台, 还包含了虚拟仿真实验平台。本教学团队 近年来在这两类平台的建设方面均取得了标志性的建设成果。

$\mathrm{Bb}$ 平台是用于加强网络教学、辅助课堂教学以及提供互动交流的专业网络教学平台 ${ }^{[3]}$ 。本教学 团队在2016年将该平台引入无机化学实验教学, 为学生搭建了实验学习的第二课堂。平台为每个实 验提供了课前和课后多个学习模块。课前模块主要包含实验背景知识、实验预习视频、小测验等, 不仅丰富了实验相关的理论知识, 更将预习过程从单纯的文字学习提升至视频学习, 为学生在课前 提供了重点实验操作的学习资料, 大大提升了预习效果及课堂实验操作规范性。课后学习板块主要 包含实验报告的提交、问题讨论等内容, 方便实验报告的保存, 总结, 并为学生的答疑解惑提供了 平台。此外, 平台上还专门设立了实验不规范行为曝光栏, 以图片形式上传教学过程中的不规范实 验行为, 在引导学生形成正确实验操作及良好实验习惯方面起到了积极督促作用。

在当前的实验教学中线下实验教学依然保持主流、不可替代的地位, 但线下实验存在时间、空 间、设备、安全性等方面的局限性, 特别是新的实验技术、实验内容引入到教学当中需要克服种种

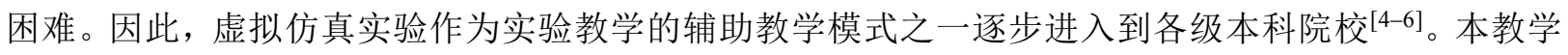
团队在 2019 年成功申报了国家级虚拟仿真教学项目 “人工光合成清洁燃料综合化学虚拟仿真实 验”，并在2020年疫情期间以及后疫情时代的本科教学中发挥了积极的作用。

此外, 我们还积极引入大学慕课平台上的优质课程资源提升学生的学习兴趣和效果。例如大连 理工大学的无机化学实验及其配套的MLabs手机端虚拟仿真实验, 作为元素化学实验的辅助教学素 材, 实验室安全模拟训练等内容也可以提升学生实验室安全素养。在使用优质的兄弟院校资源外, 我 们也在该平台上创建了多个具有本教研室特色的虚拟仿真教学实验(图1), 例如 “氨碱法制备碳酸钠” 中采用自行设计的二氧化碳发生装置, “氟化物的制备” 采用本教学团队教师的科研成果等内容, 这 些内容将与线下实验教学有机结合培养学生的创新实验思维。

\section{4 课程思政}

习近平总书记2016年在全国高校思想政治工作会议上的讲话中指出, 高等学校要坚持把立德树 人作为中心环节, 把思想政治工作贯穿教育教学全过程, 实现全程育人、全方位育人, 努力开创我 国高等教育事业发展新局面 ${ }^{[7]}$ 。如何在无机化学实验课程上深挖课程思政, 在教学过程中 “润物无

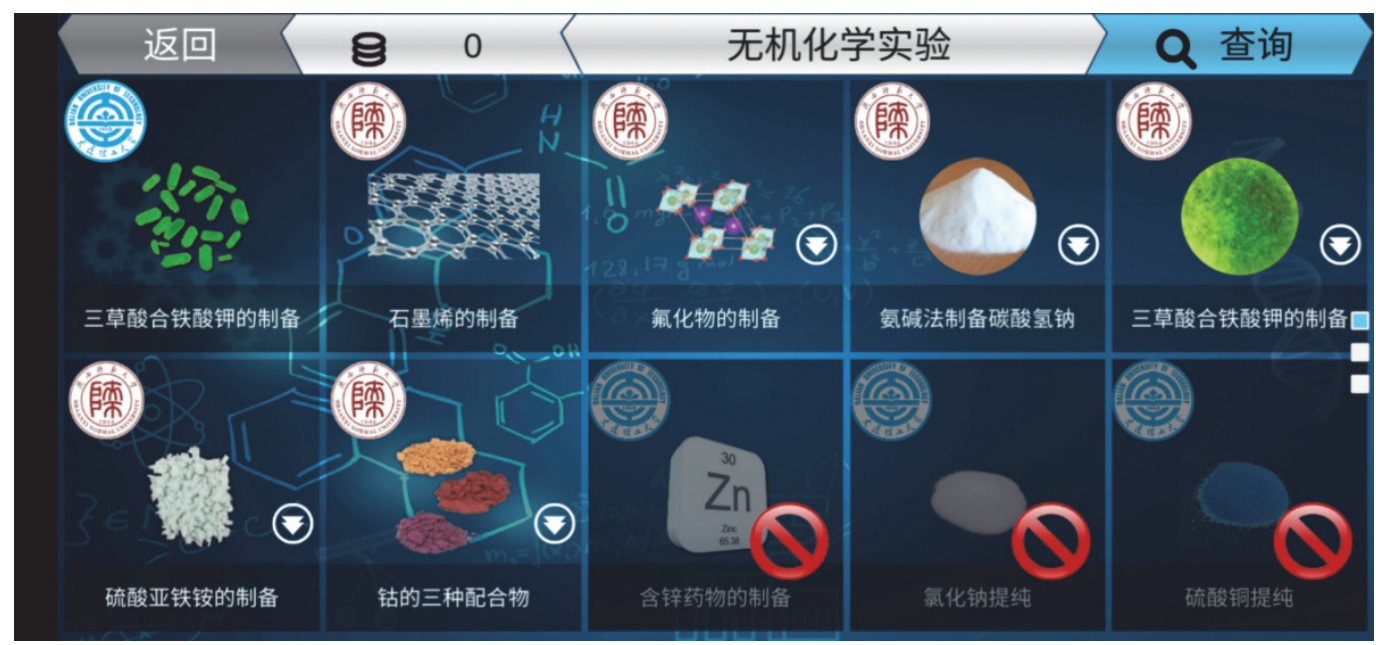

图 1 Mlabs 移动端中的陕西师范大学虚拟仿真实验界面 
声” 地将思政元素融入课堂, 是近年来各类课程改革的重点 ${ }^{[8]}$ 。教学团队通过学习讨论、实践摸索、 个人总结等方式针对每个实验进行了课程思政元素的挖掘, 总结出包括严谨的科学精神、深远的科 学视野、绿色环保意识、社会责任感、创新知识产权意识、唯物主义精神、实事求是精神等思政元 素。例如, 在 “氧化型石墨烯的制备” 实验中, 我们结合实验方案以及具体操作, 引导学生了解国 内外研究现状, 激发学生的爱国情怀、科研自信、勤于思考、勇于探索的精神, 使学生深刻体会到 中国科学家在石墨烯发展中的重要贡献和全球性科技竞争的普遍性, 进而达到实验课与专业基础课、 思政教育相结合的全方位教学, 促进学生综合素质的全面提升 ${ }^{[9]}$ 。通过思政元素的融入, 使学生在 学习实验知识的同时始终以较高的精神追求为前提, 从潜意识里努力成为一名品德高尚且志向高远 的化学人。

\section{5 实验教材与教学文章}

在不断推进以上改革方向的同时, 我们对改革成效也及时进行总结, 做到改革与总结并行, 这 样才能及时发现问题、解决问题, 实现改革的持续创新。其中, 在实验教材方面, 我们在 2015 年出 版了无机化学实验教材及配套参考书(图2)。《无机化学实验》教材中更新了包括MOFs材料、氧化型 石墨烯、苂光粉、阻燃材料等基于团队教师科研工作进展的材料制备实验。《无机化学实验手册》与 教材配套使用, 增加了相关实验的背景知识、实验操作关键点、思考题答案等内容, 便于教师和学 生快速掌握实验重点内容。此套教材在近 5 年的教学实践中取得了较好的教学效果, 学生对实验的内 容掌握更加精准, 同时由于提供了实验操作的关键点, 使教学实验成功率大大提升。
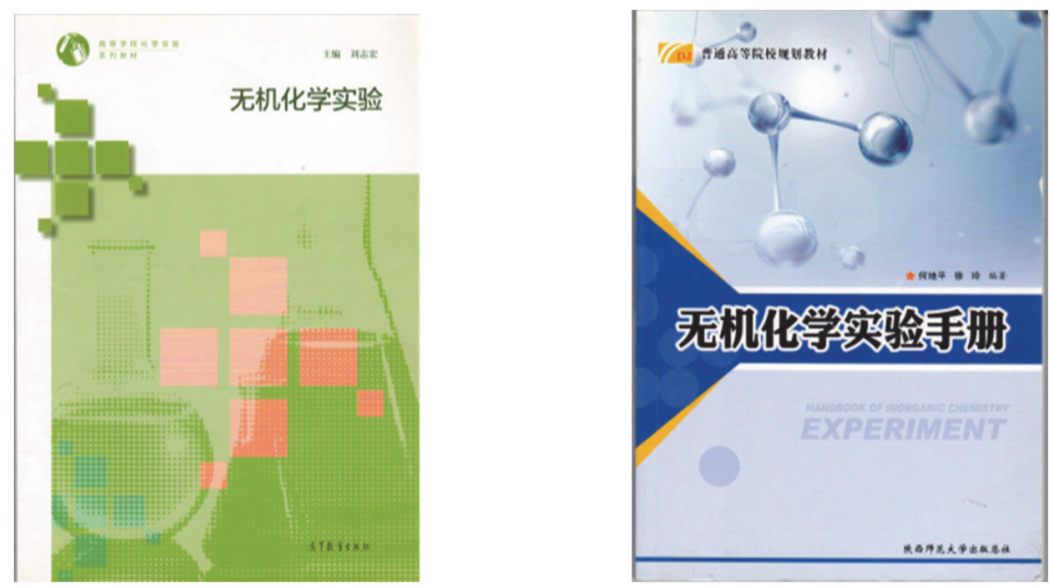

图2 主编教材及参考书— 《无机化学实验》及《无机化学实验手册》

除了教材更新之外, 对于实验教学的点滴改进, 我们均以教学论文的形式进行总结。近五年来

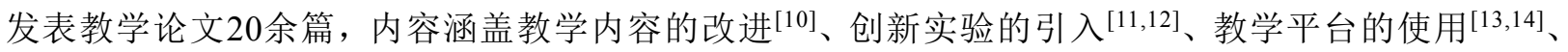

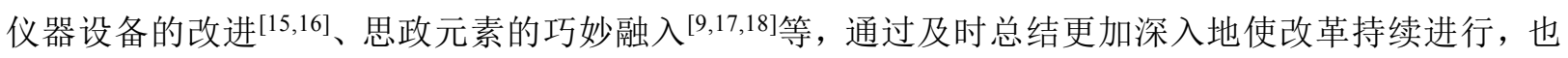
为兄弟院校的相关教学提供了一定的参考。

\section{3 典型教学实例}

以上改革要点在无机化学实验教学中均得到了体现, 典型的实验教学流程如图3所示, 一般包含 课前预习、课堂教学、实验操作以及报告及评价几部分。

下面以线下线上混合实验教学 “二氧化碳分子量的测定” 为例具体阐述。“二氧化碳分子量的测 定” 是将理论与实践结合的典型实验。实验运用到的理论包括阿伏伽德罗定理、克拉伯龙方程以及 二氧化碳制备的化学原理等, 学生需要掌握的实验技能包括气体的制备、纯化以及分析天平的使用, 下面具体阐述该课程的实施过程。 


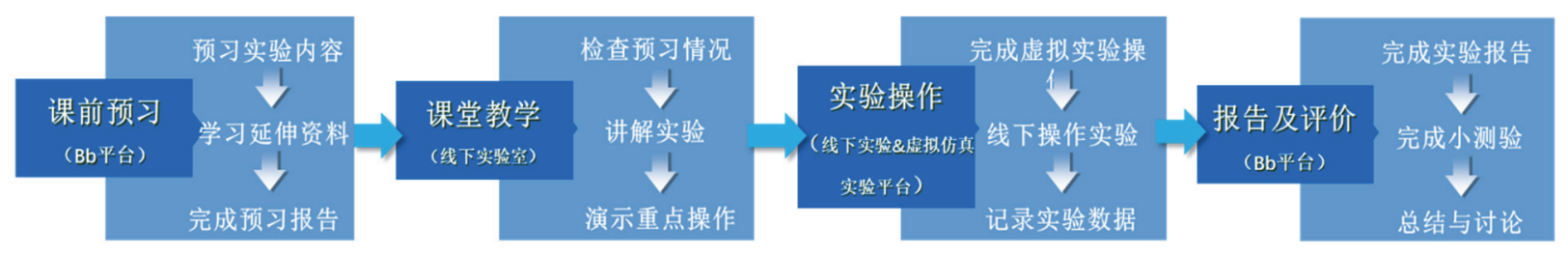

图 3 典型实验教学流程图

\section{1 课前预习}

课前预习学生将依托教材内容以及 $\mathrm{Bb}$ 平台上资料展开。如图4所示, 在平台视频资料文件夹中的 实验基本操作中提供 “分析天平的使用” 小视频, 在课程实验预习视频中提供 “二氧化碳分子量的 测定” 小视频, 使学生在课前就可通过直观的视频演示了解本次课程重点实验操作。同时, 预习完 成后将下载实验报告模板, 完成实验报告的预习部分, 包含实验目的、实验原理、实验步骤以及思 考题。该过程充分利用网络教学平台及多媒体资源, 实现预习过程不拘泥于书本、文字等固定形式, 从而使学生在实验前通过反复观看视频充分熟悉分析天平的使用及其气体发生装置的具体结构及搭 建注意事项, 大大提高了实验效率及成功率。同时, 个性化实验报告模板的提供使学生能将更多精 力放在知识点的掌握, 提高了其学习效率。

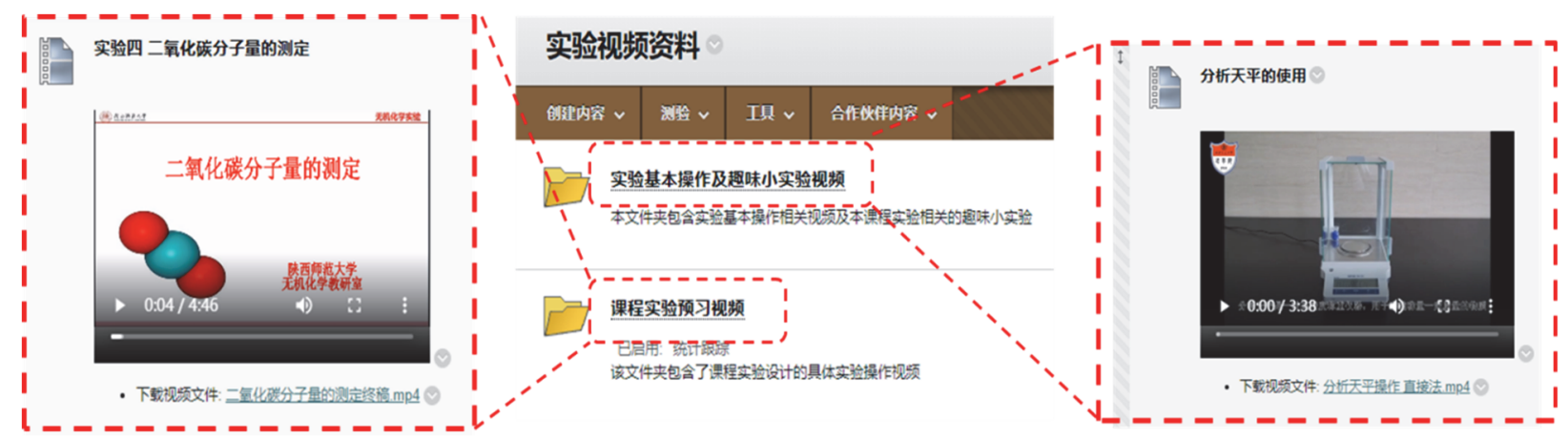

图4 Bb平台上 “二氧化碳分子量的测定” 预习视频资料

\section{2 课堂教学及实验操作}

实验课堂教学环节包含预习情况检查、实验讲解以及重点操作演示。其中, 预习情况的检查可 通过多种形式。例如, 该实验中 “阿伏伽德罗定理” 及 “克拉伯龙方程” 的定义、方程表述形式、 使用条件等采用课堂问答形式, 考查学生对理论知识的掌握情况; 分析天平的使用采用翻转课堂模 式, 让学生独立进行天平使用的演示和讲解, 并鼓励其他同学进行检验和补充, 充分调动了学习积 极性; 此外, 在讲述新型装置的正确搭建方式时, 置入雨课堂小问答环节, 检验整体学生对气体吸 收瓶结构的正确理解以及对气体发生装置搭建的注意事项的掌握情况。实验讲解努力通过图文并茂 的课件形式将重点知识和操作呈现给学生。讲授过程从课题的引入到原理的分析再到步骤剖析, 并 重点讲解了该实验中我院教师设计发明的新型气体发生装置(图5), 鼓励大家对实验装置从 “拿来就 用” 逐步转变为不断质疑、不断探索的创新模式。同时, 以此为思政出发点, 通过教师照片、专利 证书等直观图片的展示激发大家的自信心，使学生产生了对具有自主知识产权实验装置的自豪感， 无形中将民族自豪感、创新思维等思政元素融入课堂。

实验安全培训一直以来是实验教学的重中之重, 然而传统的基于文字知识的教学形式难以使学 生在发生紧急情况时快速响应。在本教学案例中, 浓硫酸的使用和浓酸灼伤的处理方式一直是教学 安全的重点, 我们结合线上虚拟仿真实验练习来完成该部分教学, 以期达到更好效果。学生将在教 
师课堂授课完成后、实验操作开始前进行虚拟仿真的练习。图6展示的是手机端MLabs虚拟仿真体系 的界面, 学生将从该APP中进入 “突发机应急事件处理” 模块, 并正确完成 “浓酸灼伤” 的突发事件 处理步骤。该过程以三维动画形式推动学生完成虚拟的浓酸灼伤处理过程, 较文字描述更直观, 也 更有效地保证了学生在遇到突发事件时的处理能力。完成该步骤后, 学生即开始实验过程, 完成实 验并记录数据。
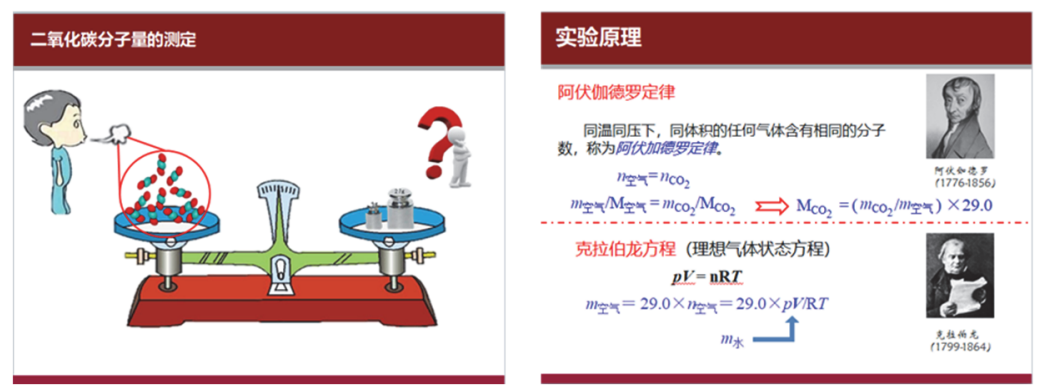

\section{实验步骤}

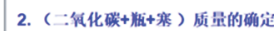

2.1 : 氮化碳的制备

$2 \mathrm{HCl}+\mathrm{CaCO}_{3}=\mathrm{CO}_{2} \mathrm{~T}+\mathrm{CaCl}_{2}+\mathrm{H}_{2} \mathrm{O}$

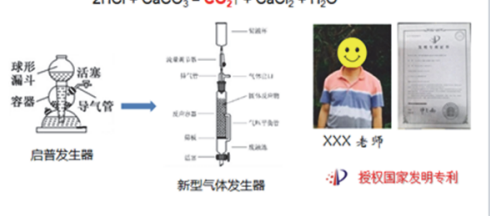

图5 课堂教学部分课件内容: 引题(左)、实验原理(中)、气体发生装置讲解(右)

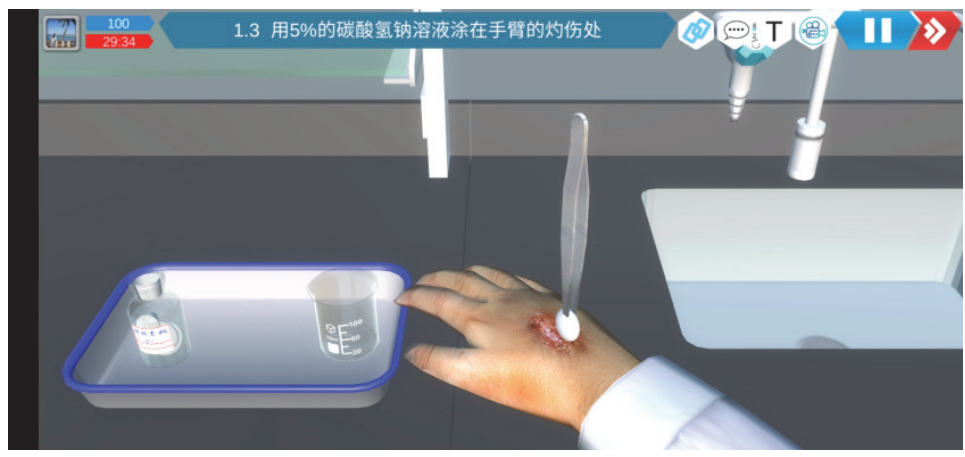

图6 MLabs虚拟仿真实验 “浓酸灼伤” 实验界面

\section{3 报告及评价}

课堂教学完成后, 学生将基于实验内容完成实验报告的实验现象、数据记录、数据处理等内容, 现象包括二氧化碳气体产生的现象, 雉形瓶充满的检验现象等, 数据包括瓶+空气质量、二氧化碳 + 瓶质量、瓶+塞子质量等, 数据处理包含计算得到的二氧化碳分子量以及二氧化碳分子量百分误差 等。学生将已完成的实验报告提交至 $\mathrm{Bb}$ 平台相应模块, 同时在线完成二氧化碳分子量的小测验。 教师可在线批改报告, 并查看测验成绩。实验报告成绩及小测验成绩将并入最后期末成绩。同时, 该实验在 $\mathrm{Bb}$ 平台上开辟了讨论版, 要求学生结合本次实验中气体发生装置的使用体会, 在讨论区发 表使用心得, 包括新型气体发生装置与启普发生器的区别? 我们的优势在哪里? 如果让你来设计, 该装置还有哪些地方可以进行改进? 这些内容都激发了学生对实验的再次反思, 有利于其创新思维 的培养。鉴于教学平台在知识传播和教学讨论方面的重要性, 学生在网络平台上的活跃度也是最终 实验成绩的重要参考内容。

\section{4 结语}

无机化学实验课程的改革关乎化学类学生培养的基石, 我们根据教学需求重新梳理了教学内容, 将经典教学内容与创新实验内容相结合, 教学模式从单一的线下教学发展成为线上线下混合教学, 将考核模式做到与具体教学内容相配套, 在教学中积极融入思政内容, 及时总结教学过程及效果。 在以 “二氧化碳分子量” 的教学案例中, 线上线下混合式教学模式的使用、新型教学手段包括雨课 
堂的使用、虚拟仿真实验的引入都充分激发了学生学习的积极性, 取得了良好的教学效果, 同时创 新思维、民族自豪感等思政元素的巧妙引入, 也为培养具有创新理念的高素质化学人才进行了探索。 无机化学实验课程改革需要长期坚持和持续推进, 我们立足于快速发展的科技时代, 应紧跟时 代发展的脚步, 积极吸取兄弟院校的教学经验, 取长补短, 不断总结, 不断质疑, 才能更进一步提 升无机化学实验的课程育人价值, 推进课程质量文化的进步。

\section{参 考 文 献}

[1] 马骁飞, 马亚鲁, 高洪苓. 大学化学, 2021, 36 (2), 2001061.

[2] 杨惠芳, 董维超, 任书霞, 郭献章. 实验技术与管理, 2021, 36 (5), 204.

[3] 陈亚苻, 苗秀秀, 王长号, 张颖, 雒婷雯. 大学化学, 2019, 34 (3), 5 .

[4] 杜静, 赵温涛, 冯霞, 马骁飞. 大学化学, 2021, 36 (1), 2005078.

[5] 姜艳红, 崔承毅, 秦晓梅, 陈景, 周晓丹, 马驰, 王开宇. 工业和信息化教育, 2019, No. 10, 1.

[6] 姜文风, 张永策, 纪敏, 田福平, 宿艳. 实验技术与管理, 2020, 37 (11), 139.

[7] 习近平. 全国高校思想政治工作会议上的讲话. 新华网. [2016-12-08]. http://www.xinhuanet.com//politics/2016-12/08/c_1120083340.htm

[8] 张玉荣, 袁耀峰. 大学化学, 2021, 36 (2), 2007057.

[9] 徐玲, 魏恒伟, 魏灵灵, 马艺, 王晓明, 焦桓. 大学化学, 2021, 36 (3), 1912042.

[10] 魏灵灵, 张可嘉, 李淑妮. 大学化学, 2020, 35 (1), 64.

[11] 魏灵灵, 焦桓. 大学化学, 2020, 35 (4), 112.

[12] 薛东旭, 郭新爱, 张玉风, 魏文琪, 白俊峰. 化学教育(中英文), 2020, 41 (14), 53.

[13] 马艺, 张伟强, 薛东旭, 张航, 顾泉, 郑浩铨, 翟全国, 高玲香. 大学化学, 2020, 35 (5), 229.

[14] 魏灵灵, 徐玲, 魏恒伟, 张永策, 胡涛, 崔沝, 焦桓. 大学化学, 2020, 35 (5), 236.

[15] 马艺, 魏恒伟, 张可嘉, 何地平, 刘环环, 尹志广, 付渝, 焦桓. 化学教育(中英文), 2020, 41 (10), 26.

[16] 徐玲, 胡定芳, 魏恒伟, 何地平, 焦桓. 教育教学论坛, 2020, No. 15, 382.

[17] 李淑妮, 翟全国, 蒋育澄, 胡满成. 大学化学, 2018, $33(3), 36$.

[18] 王红艳, 王凯, 焦桓, 刘志宏. 大学化学, 2021, 36 (3), 2007034. 